\title{
Development of Compact Cs/Cc Corrector with Annular and Circular Electrodes
}

Tadahiro Kawasaki ${ }^{1}$, Ryuji Yoshida ${ }^{1}$, Takeharu Kato ${ }^{1}$, Tsunenori Nomaguchi ${ }^{2}$, Toshihide Agemura ${ }^{2}$, Tetsuji Kodama ${ }^{3}$, Masahiro Tomita ${ }^{4}$, and Takashi Ikuta ${ }^{5}$

${ }^{1 .}$ Nanostructures Research Laboratory, Japan Fine Ceramics Center, Nagoya, Japan

${ }^{2 .}$ Hitachi High-Technologies, Hitachinaka, Japan

3. Graduate School of Science \& Technology, Meijo University, Nagoya, Japan

4. Vacuum Device Ltd., Mito, Japan

${ }^{5 .}$ Faculty of Engineering, Osaka Electro-Communication University, Neyagawa, Japan

The spherical aberration (Cs) correction is indispensable to improve the spatial resolution in the electron microscopes. Some types of Cs correction devices have been proposed and developed, and the Cscorrectors consisted of multi-pole lenses have successfully realized sub-angstrom resolution in (S)TEMs [1-2]. However, these correctors require complex control of multiple optical components with high accuracy and stability. They also demand reconfiguration of the microscope columns to insert rather large additional components, resulting in huge cost. In order to solve these problems, Ikuta had proposed a very simple and compact Cs-corrector with axially-symmetric electrostatic-filed formed between annular and circular electrodes [3-4], as schematically shown in Fig. 1(a). We called it "ACE corrector" (the Cscorrector using Annular and Circular Electrodes). Furthermore, this simple device has an additional capability to reduce the effect of the chromatic aberration $(\mathrm{Cc})$. In the present paper, we report the principle of $\mathrm{Cs} / \mathrm{Cc}$ correction and preliminary results of the ACE corrector in simulations and experiments.

Figure 1(b) shows the principle of the spherical aberration correction with the ACE corrector from the viewpoint of the geometrical optics. In this figure, an example of equipotential lines of the electrostatic field is drawn, which is obtained by numerical simulation of 3D field. Electrons going through the field around the circular electrode from left- to right-hand side are a little focused, meaning that the circular electrode acts as a convex lens. In contrast, the electron trajectories are spread around the annular electrode, corresponding to a concave lens. They indicate that the electrostatic field formed by the ACE corrector can provide the compound lens effect of the convex and concave lenses, and totally they create the negative Cs, though the effective area is restricted to be in the off-axis by the annular slit.

The chromatic aberration can be compensated by another principle. In a case of Cs-corrected system, normal convergent beam is focused toward a single point in 3D real space, as shown in Fig. 2(a). On the other hand, hollow-cone-shaped convergent beam formed by using an annular slit does not provide focused intensity distribution but elongated one along the optical axis, as shown in Fig. 2(b). This means that the depth of focus (DoF) is extended by the annular electrode in the ACE corrector. Then, focal spread caused by the chromatic aberration does not affect so strongly to image quality. This effect increases with decreasing the width and the diameter of the annular slit.

Figures 3(a) and (b) show simulation results of graphs of residual Cs and Cc values vs applied voltage between the two electrodes. Here, the Cs is reduced linearly with increasing the voltage and can be zero by applying appropriate voltage, $10.7 \mathrm{~V}$ in the present case. In contrast, the $\mathrm{Cc}$ value created by the electrostatic field is almost zero, as shown in Fig. 3(b). This indicates that the chromatic aberration can be compensated by not the electrostatic field but the annularly focusing effect. 


\section{References:}

[1] M. Haider et al, Optik 99 (1995), p. 167.

[2] O. L. Krivanek et al, Inst. Phys. Conf. 153 (1997), p. 35.

[3] T. Kawasaki et al, Suf. Int. Anal 48 (2015), p. 1160.

[4] T. Kawasaki et al, PCT/JP2016/053691

(a)

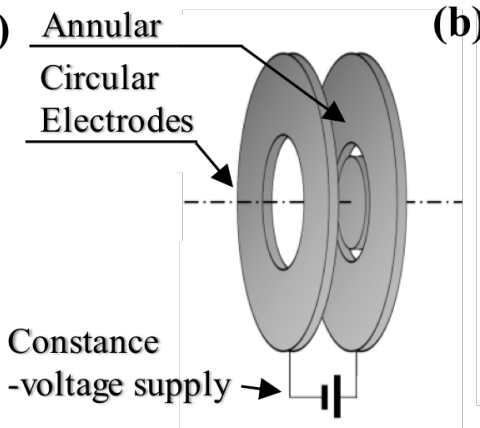

(b)

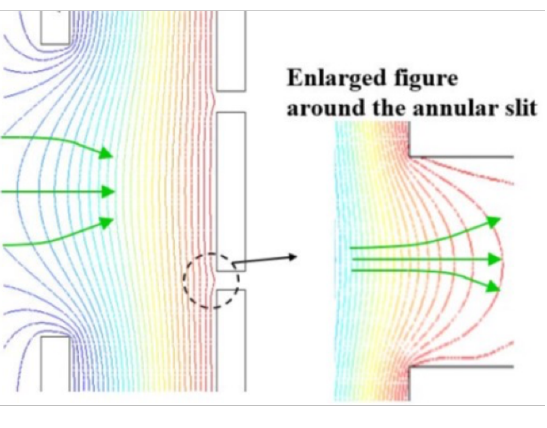

Figure 1. Schematic illustrations of (a) configuration of the ACE corrector and (b) equipotential lines inside.
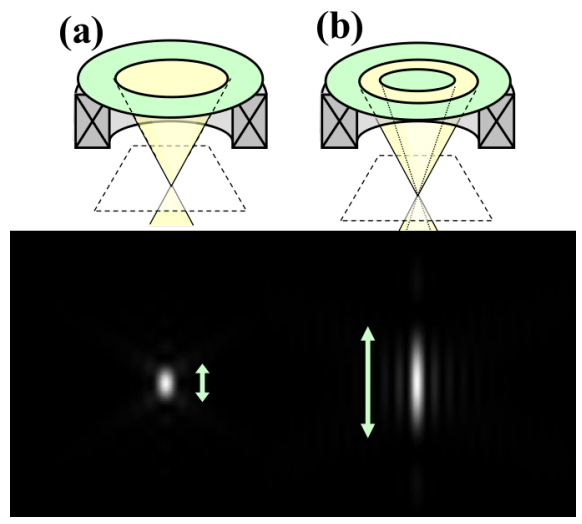

(b)
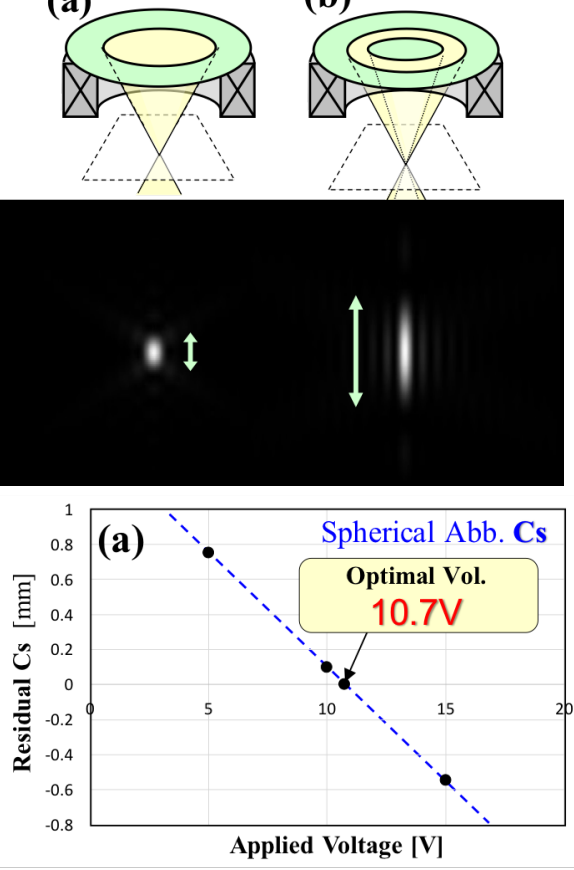

Figure 3. Graphs of (a) Cs and (b) Cc values vs applied voltages
Figure 2. Depth of focus in (a) normal and (b) hollow-cone-shaped convergent beams. 